\title{
SIMULATIONS OF THE ESA PLANCK HFI BEAMS OF POLARIZED AND MULTI-MODE HORNS
}

\author{
Vladimir B. Yurchenko ${ }^{1}$, John A. Murphy ${ }^{1}$ and J.-M. Lamarre ${ }^{2}$ \\ ${ }^{1}$ Experimental Physics Department, National University of Ireland, \\ Maynooth, Ireland \\ <v.yurchenko@may.ie> \\ ${ }^{2}$ Observatoire de Paris, 61 Av. de l'Observatoire, 75014, \\ Paris, France
}

\begin{abstract}
We report on rigorous physical optics simulations of the ESA PLANCK dualreflector telescope being designed for measuring the temperature anisotropies and polarization characteristics of the Cosmic Microwave Background. Both polarized mono-mode and non-polarized multi-mode beams covering the frequency range from $100 \mathrm{GHz}$ to $857 \mathrm{GHz}\left(D / \lambda_{\min }-5000\right)$ are computed by using a specially developed ultra-fast physical optics code propagating the source field mode-by-mode from the apertures of profiled corrugated horns simulated by the scattering matrix approach. Polarization characteristics of the broad-band incoherent beams have been computed for the purpose of the analysis of systematic errors in polarization measurements.
\end{abstract}

Keywords: ESA PLANCK Surveyor, CMB radiation, HFI instrument, PO simulations, polarization systematic errors.

\section{INTRODUCTION}

The ESA PLANCK Surveyor is the deep-space satellite being designed for the accurate measurements of temperature anisotropies and polarization characteristics of the cosmic microwave background (CMB). The satellite will carry a dual-reflector multi-beam telescope equipped with two focal plane instruments (the Low-Frequency and High-Frequency Instruments) for detecting the radiation in a wide frequency range extending from $30 \mathrm{GHz}$ to $1000 \mathrm{GHz}$ [1].

The High-Frequency Instrument (HFI) will operate in six frequency channels centered at 100, 143, 217, 353,545 and $857 \mathrm{GHz}$. The HFI consists of 36 corrugated horn antennas feeding cryogenically cooled bolometric detectors. Channels operating at 100, 143, 217 and $353 \mathrm{GHz}$ use diffraction-limited mono-mode quasi-Gaussian horns. Half of these channels will be used for polarization measurements by utilizing polarization-sensitive micromesh bolometers developed by Turner et al. [2]

The higher frequency channels ( 545 and $857 \mathrm{GHz}$ ) are non-polarized and incorporate non-diffractionlimited profiled multi-mode horns. All the HFI horns are broad band, with the bandwidth being about $30 \%$ of central frequency. The horns are specifically designed to meet extremely constrain requirements on both the primary mirror edge taper $(\sim 25-30 \mathrm{~dB})$ and the angular resolution on the sky (about 5 arcminutes at the frequencies of $217-857 \mathrm{GHz}$ ).
Since both the temperature anisotropy $\varepsilon=\delta T / T$ and the degree of polarization $d$ of the CMB radiation are extremely small $\left(\varepsilon-10^{-5}\right.$ and $\left.d<10^{-6}\right)$, the $\mathrm{CMB}$ measurements require an exquisite accuracy of the instrument and a tight control of possible sources of systematic errors. To achieve the goal, thorough simulations and testing of the instrument are needed at every stage of the design.

Optical simulations are particularly challenging because of multi-beam dual-reflector geometry of the telescope, significant refocus of elliptical mirrors, rather large primary mirror having projected diameter $D=1.5 \mathrm{~m}\left(D / \lambda_{\text {min }}-5000\right)$, strict requirements on the accuracy and, generally, multi-mode structure of the feed horn antenna fields. Physical optics (PO) is the most appropriate technique for this kind of simulations. Conventional software cannot, however, cope efficiently with the electromagnetic problem of this size.

As an alternative, a special ultra-fast PO code has been developed for the PLANCK simulations [3, 4]. It allowed us to perform rigorous $\mathrm{PO}$ simulations of the main beams of the telescope in a few minutes for mono-mode HFI beams at a single frequency and in half an hour for the complete broad-band polarizationaveraged multi-mode beams. Available comparisons of the results with much more resource-consuming GRASP8 simulations prove the accuracy and the advantages of this approach.

The aim of this paper is to summarise the results of our simulations of the ESA PLANCK HFI beams and to consider the implications of the beam imperfections on systematic errors of polarization measurements. 


\section{Simulations Of THE HORN Field}

The electric field at the aperture of corrugated horns has been simulated by the scattering matrix approach. The effective modes of the electric field at the horn aperture, $\mathbf{E}_{n m}$, are represented via the canonical TETM modes $\varepsilon_{n j}$ of a cylindrical waveguide as follows

$$
\mathbf{E}_{n m}(\rho, \varphi)=\sum_{j=1, \ldots, 2 M} S_{n m j} \varepsilon_{n j}(\rho, \varphi)
$$

where $S_{n m j}$ is the scattering matrix computed by Gleeson et al. [5] for each particular horn at various frequencies $f$ ( $S_{n m j}$ is used as an input in this work), $n=0,1, \ldots, N$ is the azimuthal index and $m, j=1,2, \ldots, 2 M$ are the radial indices accounting for both the TE $(m, j=1, \ldots, M)$ and TM $(m, j=M+1, \ldots, 2 M)$ modes.

Spatial structure of the $\mathbf{E}_{n m}$ aperture modes is essentially different from the structure of canonical modes $\varepsilon_{v j}$. It depends on the horn design and varies with varying the frequency within the bandwidth of the channel.

Notice that the terms TE and TM in application to $\mathbf{E}_{n m}$ (unlike $\varepsilon_{n j}$ ) are mere notations since, generally, none of the $\mathbf{E}_{m m}$ modes is perfectly transverse. Notice also that the total power of the horn field is the sum of powers of $\mathbf{E}_{n m}$ modes.

Fig. 1 shows the power contribution of different $\mathbf{E}_{n m}$ modes to polarized mono-mode beams HFI-143 and non-polarized multi-mode beams HFI-545. In multi-mode beams, both the power contribution and the shape of $\mathbf{E}_{n m}$ modes vary significantly within the bandwidth, with many modes being rejected at the lower frequency edge.

In case of mono-mode horns, all the aperture modes have the same shape and effectively sum up to a single mode which is of one unit of total power, almost perfectly Gaussian and linearly polarized on the hom aperture. Broad-band and mono-frequency far-field power patterns of the HFI-143 and HFI-545 horns are shown in Fig. 2.

To minimize polarization errors due to mismatch of different beams, the pairs of orthogonal polarization channels have been incorporated into the same horn by using the polarization sensitive bolometers [2]. In this case, the difference of power patterns of two channels is minimal (typically, less or about $1 \%$ ). It arises only due to minor asymmetry of the polarized modes propagating through the horn and a slight difference in propagation of different polarizations along the same path via the telescope (the mismatch of different beams is much greater and depends on the horn location in the focal plane).

\section{Simulations of Multi-Mode Beams}

Simulations of multi-mode beams require the propagation of each mode for a few times with different polarization angle for proper averaging of polariza-

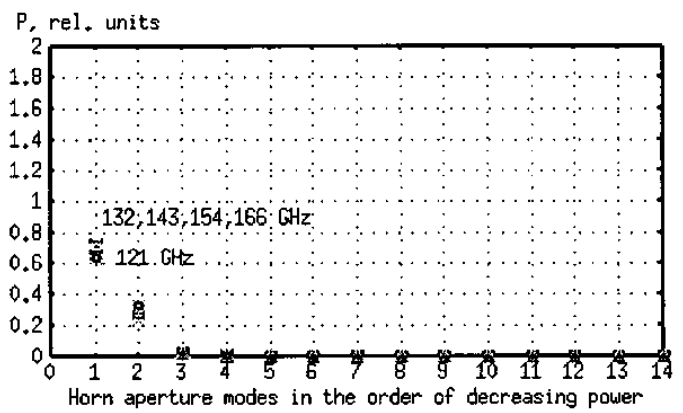

a)

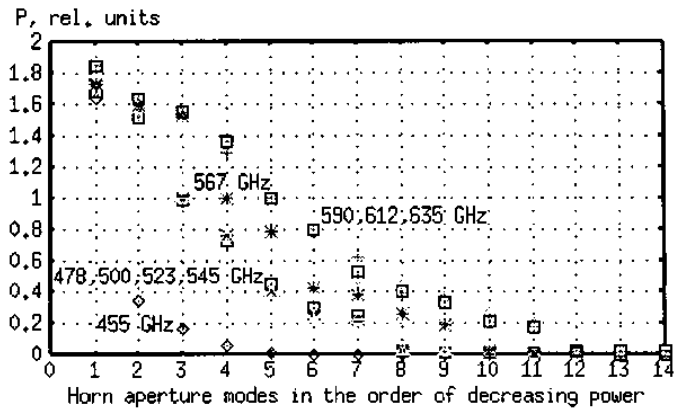

b)

Fig. 1. Contribution of $\mathbf{E}_{n m}$ modes to (a) $\mathrm{HFl}-143$ and (b) HFI-545 beams in units of power of a single polarized mode

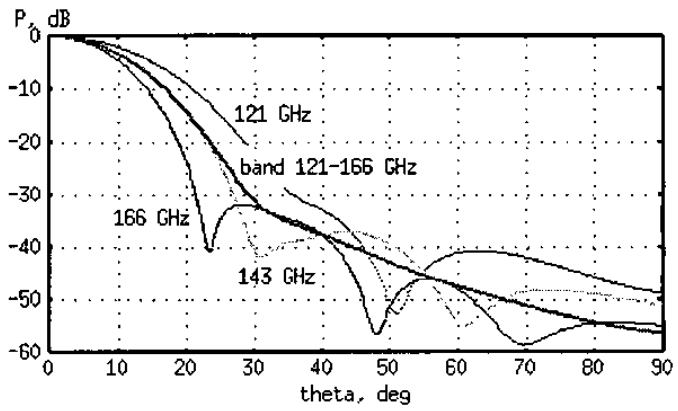

a)

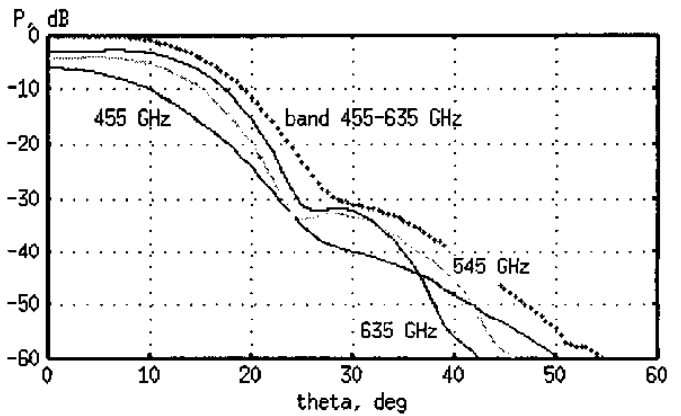

b)

Fig. 2. Broad-band and mono-frequency far-field power patterns of (a) HFI-143 and (b) HFI545 horns 


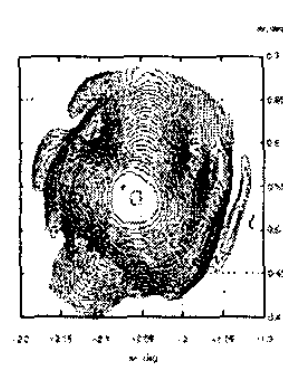

a)

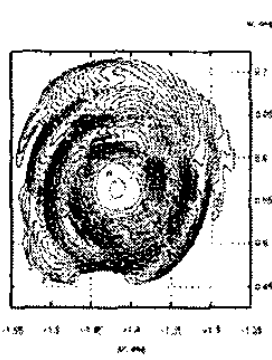

c)

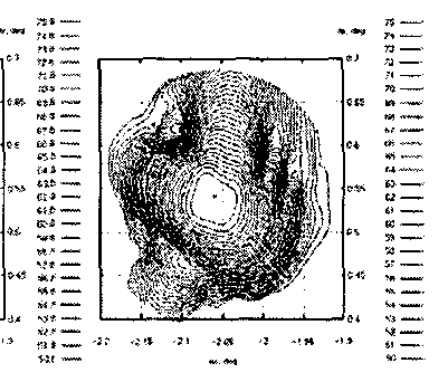

b)

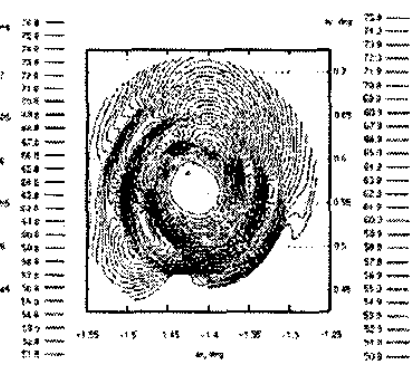

d)
Fig. 3. Power patterns of $(a, c)$ mono-frequency and (b,d) broad-band telescope beams from the multi-mode horns $(a, b)$ HFI-545-1 and $(c, d)$ HFI-545-2 computed by the scattering matrix approach

tion. The reason is that, unlike the field of a conical or Gaussian horn, the modes of the profiled horns are not axially symmetric and the mode pattern in the sky depends on the polarization angle on the horn aperture. Also, because of broad frequency bands $(\sim 30 \%)$, averaging over frequency is needed as well.

Fig. 3 shows the mono-frequency $(f=545 \mathrm{GHz}$ ) and broad-band ( $f=455-635 \mathrm{GHz}$ ) power patterns of the telescope beams from the profiled corrugated horns HFl-545-1 and HFI-545-2 computed by the scattering matrix approach with averaging over polarization angle. Each hom is placed at the best refocus providing both the maximum gain and the best resolution of the telescope. The best refocus is found by repeated PO simulations of the broad-band beams with different locations of the horn. For these multi-mode horns with the aperture radius $a=4.298 \mathrm{~mm}$, the best refocus corresponds to the effective focal centre located at $R_{C}=5.0 \mathrm{~mm}$ inside the horn as measured from the horn aperture.

The beams in Fig. 3 are computed with the horns optimized for the required angular resolution of the telescope (the full beam width on the sky $W=5$ arcminutes) when satisfying the edge taper requirements on the primary mirror. The distortion of the beams at this level of resolution is mainly due to coma (aberrations of the wider beams are mainly due to astigmatism). Each beam has a well-defined flat top of the angular diameter $W=4.8$ arcminutes when measured at the level of $-3 \mathrm{~dB}$ which appears to be just the edge of the flat top. At the level below $10 \mathrm{~dB}$, the beams, however, are rather complicated because of significant aberrations of the telescope. The aberrations are different for different modes and vary significantly when sweeping the frequency across the bandwidth of the frequency channel.

\section{Simulations of Polarized Beams}

Polarized mono-mode beams of channels $f=100$, 143,217 and $353 \mathrm{GHz}$ are produced by profiled corrugated horns rather similar to multi-mode ones. These horns are also simulated in a general way as multi-mode ones. However, due to Gaussian-like profile, selection of modes and sensitivity of the bolometers to only one linear polarization, the aperture field of these horns consists of just two effective modes $\mathrm{TE}_{1 \mathrm{t}}$ and $\mathrm{TM}_{11}$ (Fig. 1,a) producing almost the same pattern on the sky both in terms of power and polarization.

Because of these properties, the aperture field of the polarized horns can be well simulated by a clipped Gaussian source field with some curvature of the wave front. This model allowed us to find the required orientation of the polarization vector on the aperture of each horn by computing the orientation of the polarization ellipse on the axis of the coherent telescope beam at the central frequency of each channel [4].

More rigorous approach requires the scattering matrix simulations with averaging of the polarization data over all the contributing modes and over all the frequencies of incoherent beam. The averaging of this kind is computed by representing the polarization data in terms of Stokes parameters of the telescope beams.

We compute Stokes parameters defined with respect to the parallels and meridians of the spherical frame on the sky with the pole being the geometrical spin axis of telescope (the spherical frame of spacecraft). This definition has an advantage that the polarization data of this representation can be easily converted into the invariant E-B representation which is independent of the coordinate frame on the sky. Also, this spherical frame does not have artificial singularities in the field of view of telescope and the beam patterns are directly superimposed and compared on the sky when the telescope is spinning about its geometrical axis.

Fig. 4 shows the broad-band power patterns computed in this way for two pairs of beams, HFI-143-2a and HFI-143-4a of one pair, and HFI-217-6a and HFI217-8a of another pair. It is the beams of each pair that are directly superimposed on the sky when spinning the telescope. The patterns in Fig. 4 are plotted as projected on the plane normal to the line of sight of telescope.

Accurate polarization measurements require identical power patterns and perfect superposition of two beams on the sky. In reality, as shown in Fig. 4, the beams are distorted in different manner because of different locations of horns on the focal plane, and the 


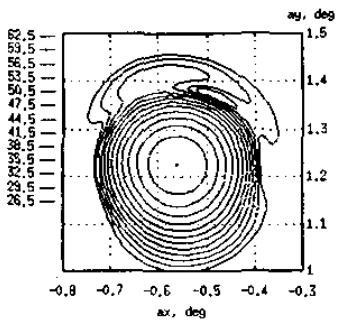

a) HFI-143-2a

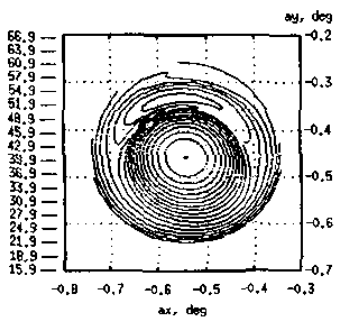

c) HFI-217-6a

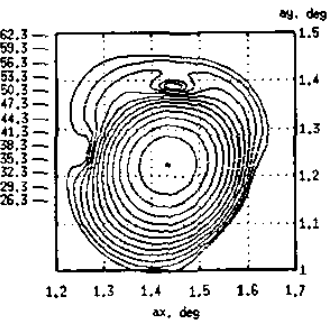

b) HFI-143-4a

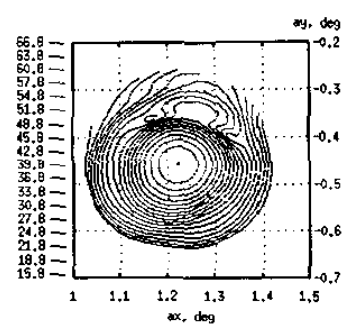

d) HFI-217-8a

Fig. 4. Power patterns of the pairs of beams to be superimposed on the sky ( $a$ with $b$ and $c$ with d) for polarization measurements when spinning the telescope (broad-band channels (a,b) $f=121-166 \mathrm{GHz}$ and (c,d) $f=182-252 \mathrm{GHz}$ )

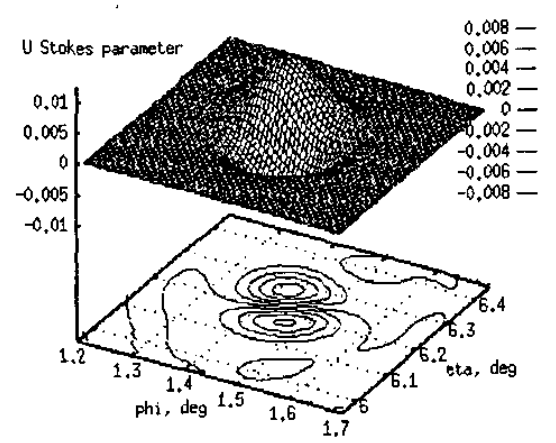

Fig. 5. The $U$ Stokes parameter pattern of the broad-band beam HFI-143-4a

difference of power patterns provides an important estimate on the level of possible systematic errors in the measurements of CMB polarization.

Fig. 5 shows the $U$ Stokes parameter pattern of the broad-band beam HFI-143-4a (the $V$ pattern is rather similar, although greater in magnitude and slightly twisted about the beam axis).

As one can see, positive and negative values of the $U$ parameter are well balanced over the beam pattern and the average value is very close to zero. It proves that the chosen direction of polarization of the horn field as found by Yurchenko [4] for a simplified model of a coherent mono-frequency quasi-Gaussian beam is really an optimum (ideally, $U$ should be zero in this polarization).

The amplitude values of both the $U$ and $V$ parameters are small enough $(|U|<1 \%$ and $|V|<4 \%$ of maximum beam intensity) that ensures a sufficiently small magnitude of the cross-polarized power $\left(P_{\text {cross }}<-30 \mathrm{~dB}\right)$ as required by the telescope specification.

Recent simulations of the HFI-100 channels show that even these rather low-frequency beams are sufficiently perfect (difference of power patterns of orthogonal polarization of the same beam is about $1 \%$ ) and the cross-polarized power is well below $-30 \mathrm{~dB}$ (e.g., for the HFI-100-3a beam, $P_{\text {cross }}<-37 \mathrm{~dB}$ ).

\section{Conclusion}

Polarized mono-mode and non-polarized multi-mode HFI beams of the ESA PLANCK telescope have been computed by physical optics propagation of the horn aperture field simulated by the scattering matrix approach. Beam patterns of Stokes parameters have been computed and the required polarization angles on the horn apertures have been found. Beam patterns have been studied for the estimate of systematic errors in polarization measurements.

\section{ACKNOWLEDGEMENTS}

This work was supported by the Enterprise Ireland Basic Research Grant and by the Ulysses Research Visit Grants 2002 and 2003.

\section{REFERENCES}

1. Tauber J. A. The PLANCK mission: overview and current status // Astrophys. Lett. Comm. 2000. Vol. 37. p. 145-150.

2. Turner A. D. et al. Silicon nitride micromesh bolometer array for submillimeter astrophysics // Applied Optics, 2001. Vol. 40. p. 4921-4932.

3. Yurchenko V. B, Murphy J. A., and Lamarre J.-M. Fast physical optics simulations of the multi-beam dual-reflector submillimeter-wave telescope on the ESA PLANCK Surveyor // Int. J. Infrared and Millimeter Waves, 2001. Vol. 22. No. 1. p. 173-184.

4. Yurchenko V. B, Murphy J. A., and Lamarre J.-M. PLANCK HFI beam simulations for polarized and multi-moded horns $/ / 25^{\text {th }}$ ESA Antenna Workshop on Satellite Antenna Technology, 18-20 September, 2002, ESTEC, Noordwijk, The Netherlands. K. van't Klooster and L. Fanchi, Eds. 2002. p. 281-286.

5. Gleeson E., Murphy J. A., Maffei B., Lamarre J.M., and Wylde R. J. Definition of the multi-mode horns for the HFI instrument on PLANCK // ibid. p. 649-655.

6. Yurchenko V.B. Measuring CMB polarization with ESA PLANCK submm-wave telescope // Experimental Cosmology at Millimetre Wavelengths: 2K1BC Workshop, Breuil-Cervinia, Italy, July 9-12, 2001. M. De Petris, P. A. Moro, and M. Gervasi, Eds. AIP Conf. Proc. 2002. Vol. 616. p. 234-238. 